\title{
Proceedings of the 2015 Symposium and Bootcamp on the Science of Security (HotSoS)
}

April 21-22, 2015, Urbana, IL, USA

HotSoS 2015

\section{Organizing Committee}

- General Chair: David Nicol, University of Illinois at Urbana-Champaign

- Web Chair: Andrea Whitesell, University of Illinois at Urbana-Champaign

- Publicity Chair: Kim Gudeman, University of Illinois at Urbana-Champaign

- Finance Chair: Wyatt Martin, University of Illinois at Urbana-Champaign

- Proceedings Chair: Zbigniew Kalbarczyk, University of Illinois at Urbana-Champaign

- NSA Liaisons: Heather Lucas and Stephanie Askins-Yannacci

\section{Technical Program Committee}

- Ehab Al-Shaer, University of North Carolina Charlotte

- Adam Aviv, United States Naval Academy

- Travis Breaux, Carnegie Mellon University

- Kevin Butler, University of Florida

- Marshini Chetty, University of Maryland

- Michael Clifford, National Security Agency

- Michel Cukier, University of Maryland

- Tudor Dumitras, University of Maryland

- Serge Egelman, University of California Berkeley

- William Enck, North Carolina State University

- Robert Ford, Florida Institute of Technology

- David Garlan, Carnegie Mellon University

- Brighten Godfrey, University of Illinois at Urbana-Champaign

- Donald Goff, Cyber Pack Ventures

- Zbigniew Kalbarczyk, University of Illinois at Urbana-Champaign

- Jonathan Katz, University of Maryland

- Stuart Krohn, National Security Agency

- Lucas Layman, University of Maryland

- Carl Landwehr, Consultant

- Sam Malek, George Mason University

- Chris Mayhorn, North Carolina State University

- Andy Meneely, Rochester Institute of Technology

- Sayan Mitra, University of Illinois at Urbana-Champaign 
- Sean Peisert, University of California Davis

- Juergen Pfeffer, Carnegie Mellon University

- Sean Smith, Dartmouth College

- Robert St. Amant, North Carolina State University

- Kevin Sullivan, University of Virginia

- Kymie Tan, National Aeronautics and Space Administration

- Adam Tagert, National Security Agency

- Aad Van Moorsel, University of Newcastle upon Tyne

- Rebecca Wright, Rutgers University

- Tao Xie, University of Illinois at Urbana-Champaign 


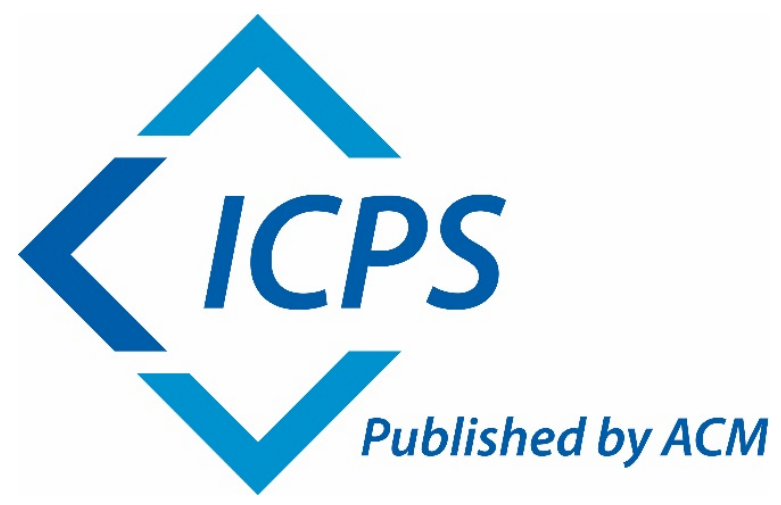

The Association for Computing Machinery, Inc.

2 Penn Plaza, Suite 701

New York, New York 10121

ACM COPYRIGHT NOTICE. Copyright (C) 2015 by the Association for Computing Machinery, Inc. (ACM). Permission to make digital or hard copies of portions of this work for personal or classroom use is granted without fee provided that the copies are not made or distributed for profit or commercial advantage and that copies bear this notice and the full citation on the first page in print or the first screen in digital media. Copyrights for components of this work owned by others than ACM must be honored.

Abstracting with credit is permitted.

To copy otherwise, to republish, to post on servers, or to redistribute to lists, requires prior specific permission and/or a fee. Send written requests for republication to ACM Publications, Copyright \& Permissions at the address above or fax +1 (212) 869-0481 or email permissions@acm.org.

For other copying of articles that carry a code at the bottom of the first or last page, copying is permitted provided that the per-copy fee indicated in the code is paid through the Copyright Clearance Center, 222 Rosewood Drive, Danvers, MA 01923.

\section{Notice to Past Authors of ACM-Published Articles}

ACM intends to create a complete electronic archive of all articles and/or other material previously published by ACM. If you wrote a work that was previously published by ACM in any journal or conference proceedings prior to 1978, or any SIG Newsletter at any time, and you do NOT want this work to appear in the ACM Digital Library, please inform permissions@acm.org, stating the title of the work, the author(s), and where and when published.

ACM ISBN: 978-1-4503-3376-4 


\section{Table of Contents}

1. Integrity Assurance in Resource-Bounded Systems through Stochastic Message Authentication Aron Laszka, Yevgeny Vorobeychik and Xenofon Koutsoukos

2. Active Cyber Defense Dynamics Exhibiting Rich Phenomena Ren Zheng, Wenlian Lu and Shouhuai Xu

3. Towards a Science of Trust Dusko Pavlovic

4. Challenges with Applying Vulnerability Prediction Models Patrick Morrison, Kim Herzig, Brendan Murphy and Laurie Williams

5. Preemptive Intrusion Detection: Theoretical Framework and Real-World Measurements Phuong Cao, Eric Badger, Zbigniew Kalbarczyk, Ravishankar Iyer and Adam Slagell

6. Enabling Forensics by Proposing Heuristics to Identify Mandatory Log Events Jason King, Rahul Pandita and Laurie Williams

7. Modelling User Availability in Workflow Resiliency Analysis John C. Mace, Charles Morisset and Aad van Moorsel

8. An Empirical Study of Global Malware Encounters Ghita Mezzour, Kathleen M. Carley and L. Richard Carley

9. An Integrated Computer-Aided Cognitive Task Analysis Method for Tracing Cyber-Attack Analysis Processes Chen Zhong, John Yen, Peng Liu, Robert Erbacher, Renee Etoty and Christopher Garneau

10. All Signals Go: Investigating How Individual Differences Affect Performance on a Medical Diagnosis Task Designed to Parallel a Signal Intelligence Analyst Task Allaire Welk and Christopher Mayhorn

11. Detecting Abnormal User Behavior Through Pattern-mining Input Device Analytics Ignacio X. Dominguez, Alok Goel, David L. Roberts and Robert St. Amant

12. Understanding Sanction under Variable Observability in a Secure, Collaborative Environment Honying Du, Bennett Narron, Nirav Ajmeri, Emily Berglund, Jon Doyle and Munindar Singh

13. Measuring the Security Impacts of Password Policies Using Cognitive Behavioral Agent-Based Modeling Vijay Kothari, Jim Blythe, Sean W. Smith and Ross Koppel

14. Effectiveness of a Phishing Warning in Field Settings Weining Yang, Jing Chen, Aiping Xiong, Robert Proctor and Ninghui Li

15. Architecture Style for Android Security Analysis Bradley Schmerl, Jeff Gennari and David Garlan

16. PREDICT: An Important Resource for the Science of Security Charlotte Scheper and Susanna Cantor

17. Characterizing Complexity of Highly-Configurable Systems with Variational Call Graphs Gabriel Ferreira, Christian Kaestner, Juergen Pfeffer and Sven Apel

18. Towards Quantification of Policy Complexity and Its Application to Firewall Policies Haining Chen, Omar Chowdhury, Ninghui Li, Jing Chen and Robert Proctor

19. Packer Classifier Based on PE Header Information Qiao Jin, Jiayi Duan, Michael Bailey and Shobha Vasudevan

20. Detecting Insider Threats in Software Systems using Graph Models of Behavior Paths Hemank Lamba and Juergen Pfeffer

21. Quantitative Security Metrics with Human in the Loop Mohammad Noureddine, Ken Keefe, William H. Sanders and Masooda Bashir 
22. Exploring Expert and Novice Mental Models of Phishing Olga Zielinska, Allaire Welk, Christopher Mayhorn and Emerson Murphy-Hill

23. A Security Practices Evaluation Framework Patrick Morrison

24. Towards an Unified Security Testbed and Security Analytics Framework Phuong Cao, Eric Badger, Zbigniew T. Kalbarczyk, Ravishankar K. Iyer, Alexander Withers and Adam Slagell

25. Mismorphism: A Semiotic Model of Computer Security Circumvention Sean W. Smith, Ross Koppel, Jim Blythe and Vijay Kothari

26. Gamifying Software Security Education and Training via Secure Coding Duels in Code Hunt Tao Xie, Judith Bishop, Nikolai Tillmann and Jonathan de Halleux

27. Systematization of Metrics in Intrusion Detection Systems Yufan Huang, Huaiyu Dai and Xiaofan He

28. Optimisation of Data Collection Strategies for Model-Based Evaluation and Decision-Making Robert Cain and Aad van Moorsel 


\title{
Message from the Program Chairs
}

\author{
David M. Nicol \\ Information Trust Institute \\ University of Illinois at Urbana-Champaign \\ Urbana, IL, USA \\ dmnicol@illinois.edu
}

\section{INTRODUCTION}

The Symposium and Bootcamp on the Science of Security (HotSoS), is a research event centered on the Science of Security (SoS). HotSoS 2015 follows on the heels of HotSoS 2014, establishing what we expect will be an annual pattern for bringing together researchers in the Science of Security.

The motivation behind the study of the Science of Security is to focus on systems' security properties as fist-class objects of study. The challenges are in defining those properties precisely within some kind of modeling framework, prove theorems about those properties and how they are achieved, identify metrics and means of empirically gathering, estimating, and/or inferring them in an experimental context, design effective experiments to gather those metrics and make statistically significant inferences about them, and close the loop by validating the abstract models with experiments.

\section{PROGRAM}

HotSoS 2015 received 22 full paper submissions, from which 13 were selected by a double-blind reviewing process. Each paper submitted received at least two reviews, most papers received three. We also accepted 16 poster submissions.

The full papers are organized into four research sessions, showing both the breadth of the interest, but also the presence of more concentrated areas of current interest. :

- Architectureand Analysis.

- Socio-technical Systems.

- Human Aspects.

- Adaptivity and Dynamics.

We also organized a time devoted for interacting with the posters and their presenters.

\subsection{Invited Talks}

The program included threekeynote talks.

- Mike Reiter, Is it Science or Engineering? A Sampling of Recent Research.

- Jonathan Spring, Avoiding Pseudoscience in the Science of Security.

- Patrick McDaniel, The Importance of Measurement and Decision Making to a Science of Security.

In addition, the program included one talk based on a paper that was recognized as Best in the 2013 NSA Scientific Cybersecurity Paper Competition ${ }^{1}$ :

\footnotetext{
$1_{\mathrm{http}: / / \mathrm{cps}-\text { vo.org/node/9941 }}$
}

- Chang Liu, Michael Hicks and Elaine Shi, Memory Trace Oblivious Program Execution for Cloud Computing.

\subsection{Tutorials}

We supplemented the research program with five tutorials.

- Kathleen Carley, Carnegie-Mellon University, Social Network Analysis for Science of Security.

- Sean W. Smith, Dartmouth College and Jim Blythe, University of Southern California, Understanding and Accounting for Human Behavior.

- Munindar Singh, North Carolina State University, PolicyGoverned Secure Collaboration.

- William H. Sanders, University of Illinois at UrbanaChampaign, Security-Metrics-Driven Evaluation, Design, Development and Deployment.

- Ravishankar Iyer, University of Illinois at UrbanaChampaign, Resilient Architectures.

\subsection{IRN-SoS}

HotSoS included a workshop of the International Research Network for the Science of Security or IRN-SoS, for short. This year's workshop is led by Laurie Williams of North Carolina State University and Jeff Carver of the University of Alabama. The workshop focused on analyzing papers from the security literature to determine how completely authors describe their research methods. The annual IRN-SoS meeting (held during HotSoS) is a venue to discuss topics regarding methodology, as well as an opportunity for members to present and receive feedback on current on future projects.

\section{ACKNOWLEDGMENTS}

We thank our sponsors, including the National Security Agency, the National Science Foundation, the Army Research Office, and the University of Illinois at Urbana-Champaign for underwriting HotSoS. We also thank ACM SIGSAC for granting HotSoS in cooperation status and ACM for accepting the HotSoS proceedings for its Digital Library.

We thank the volunteer organizers as well as University of Illinois at Urbana-Champaign staff for tackling the local arrangements and other logistics for HotSoS. Most of all, we thank the authors and program committee members without whose efforts there would be no event. 